\title{
CONJUGATE LOCI IN GRASSMANN MANIFOLDS
}

\author{
BY YUNG-CHOW WONG
}

Communicated by S. Smale, September 29, 1967

1. Introduction. In the tangent space $M_{x}$ to a Riemannian manifold $M$ at the point $x$, a conjugate point $v$ is a point at which the differential of the exponential map $\exp _{x}: M_{x} \rightarrow M$ is singular. In $M$, a point $y$ is a conjugate point to $x$ if $y=\exp _{x} v$ for some conjugate point $v$ in $M_{x}$. The conjugate locus in $M_{x}$ is the set of conjugate points in $M_{x}$, and the conjugate locus in $M$ at $x$ is the set of conjugate points to $x$.

Though there are a number of general results on the conjugate locus either in $M_{x}$ or in $M$ ([4], [6, p. 59], [11], [12] and [13]), the precise nature of this locus in special Riemannian manifolds seems to be known only in a few cases, such as the sphere, the projective spaces, and some two-dimensional manifolds ([2, pp. 225-226], [9] and [10]). In the present note, we give a complete description of the conjugate locus at a point in the real, complex or quaternionic Grassmann manifolds. Besides being useful and interesting, this information will extend the range of problems recently studied by Klingenberg [8], Allamigeon [1], Green [5] and Warner [12, 13]. The conjugate locus in the tangent space to a Grassmann manifold is more complex and will be the subject of a future note.

In $\$ 2$, we describe the Schubert varieties of which the conjugate locus in a Grassmann manifold is composed. In $\$ 3$, we give some results concerning conjugate points in a Grassmann manifold. In $\$ 4$, we state our main theorem. Details and proof will be omitted. For background information, the reader is referred to the author's paper [14].

2. Some Schubert varieties (cf. [3, Chapter 4] and [7, Chapter 14]). Let $F$ be the field $R$ of real numbers, the field $C$ of complex numbers, or the field $H$ of real quaternions; $F^{n+m}$ an $(n+m)$-dimensional left vector space over $F$ provided with a positive definite hermitian inner product; $G_{n}\left(F^{n+m}\right)$ the Grassmann manifold of $n$-planes in $F^{n+m}$.

In $F^{n+m}$, let $\boldsymbol{P}$ be a fixed $p$-plane $(1<p<n+m), \boldsymbol{Z}$ a variable $n$ plane, and

$$
\begin{aligned}
V_{l} & =\{\boldsymbol{Z}: \operatorname{dim}(\boldsymbol{Z} \cap P) \geqq l\} \quad(l \geqq 0), \\
W_{l} & =V_{l} \backslash V_{l+1}=\{\boldsymbol{Z}: \operatorname{dim}(\boldsymbol{Z} \cap P)=l\} \quad(l \geqq 0) .
\end{aligned}
$$


Then it is easy to see that $V_{l}=G_{n}\left(F^{n+m}\right)$ if $l=\max (0, p-m)$, and $V_{l}$ is empty if $l>\min (n, p)$. For the remaining values of $l$, we can prove

TheOREM 2.1. Let $k$ be any integer such that

$$
\max (1, p-m+1) \leqq k \leqq \min (n, p) .
$$

(a) The subset $V_{k}$ of $G_{n}\left(F^{n+m}\right)$ is a Schubert variety

$$
(p-k, \cdots, p-k, m, \cdots, m),
$$

where $p-k$ appears $k$ times and $m$ appears $n-k$ times. The $F$-dimension of $V_{k}$ is $n m-k(m-p+k)$.

(b) $V_{k+1}$ is the singular locus of $V_{k}$.

(c) $V_{k}$ can be decomposed into the disjoint union

$$
V_{k}=W_{k} \cup W_{k+1} \cup \ldots \cup W_{\min (n, p),}
$$

where

$$
\begin{array}{rlrl}
W_{\min (n, p)} & =G_{n}\left(F^{p}\right) & & \text { if } p>n, \\
& =\{P\} & & \text { if } p=n, \\
& \approx G_{m}\left(F^{n+m-p)}\right) & \text { if } p<n,
\end{array}
$$

and each $W_{l}(k \leqq l \leqq \min (n, p)-1)$ is a "tensor" bundle whose base space is $G_{l}\left(F^{p}\right) \times G_{n-l}\left(F^{n+m-p}\right)$, whose standard fiber is the tensor product $\left(F^{n-l}\right)^{*} \otimes F^{p-l}$ of an $(n-l)$-dimensional right vector space and $a(p-l)$ dimensional left vector space, and whose group is the tensor product $G L(n-l, F) \otimes G L(p-l, F)$; the fiber of $W_{l}$ over the point $(x, y) \in G_{l}\left(F^{p}\right) \times G_{n-l}\left(F^{n+m-p}\right)$ consists of all those n-planes $\boldsymbol{Z}$ such that $\boldsymbol{Z} \cap \boldsymbol{P}$ is the fixed l-plane $\mathbf{x}$, and the projection of $\boldsymbol{Z}$ in the orthogonal complement of $\boldsymbol{P}$ in $F^{n+m}$ is the fixed $(n-l)$-plane $y$.

Two special cases are of interest to us. Let $O$ be a fixed $n$-plane in $F^{n+m}$ and $O^{\perp}$ its orthogonal complement, and let

$$
V_{\boldsymbol{l}}=\left\{\boldsymbol{Z}: \operatorname{dim}\left(\boldsymbol{Z} \cap \boldsymbol{O}^{\perp}\right) \geqq l\right\}, \quad \tilde{V}_{\boldsymbol{l}}=\{\boldsymbol{Z}: \operatorname{dim}(\boldsymbol{Z} \cap \boldsymbol{O}) \geqq l\} .
$$

It turns out that the cut locus at the point $O$ in $G_{n}\left(F^{n+m}\right)$ is $V_{1}$ (see [14, Theorem 9(b)]), and the conjugate locus at the point $O$ in $G_{n}\left(F^{n+m}\right)$ is the union of $V_{1}$ or $V_{2}$ and one of the $\tilde{V}_{l}$ 's (see $\$ 4$ ).

3. Geodesics and conjugate points in $G_{n}\left(F^{n+m}\right)$. As in [14], let $G_{n}\left(F^{n+m}\right)$ be provided with the invariant Riemannian metric $d s^{2}$ $=\sum_{i}\left(d \theta_{i}\right)^{2}$, where $d \theta_{i}(1 \leqq i \leqq n)$ are the $n$ angles between two consecutive $n$-planes in $F^{n+m}$. Then $G_{n}\left(F^{n+m}\right)$ is a complete globally- 
symmetric space. It is known that the geodesics in $G_{n}\left(F^{n+m}\right)$, when viewed as a 1-parameter family of $n$-planes in $F^{n+m}$, are characterized by the following properties: (a) All the pairs of nearby $n$-planes of this family have common angle 2-planes (some of which may degenerate into angle 1-planes), and (b) the $n$ angles between every pair of nearby $n$-planes are proportional to a fixed set of constants.

Let $O$ and $A$ be any two points in $G_{n}\left(F^{n+m}\right)$, and $\Gamma$ any geodesic segment joining $\boldsymbol{O}$ and $\boldsymbol{A}$. Then each common angle 2-plane of $\Gamma$ either coincides with or contains an angle 2-plane between $\boldsymbol{O}$ and $\boldsymbol{A}$. If $q$ (resp. $p$ ) is the number of nondegenerate angle 2-planes of $\Gamma$ (resp. between $O$ and $A$ ), so that $1 \leqq p \leqq q \leqq \min (n, m)$, then $\Gamma$ is said to be of the $(q-p+1)$ th type. Among the geodesic segments of the $(q-p+1)$ th type joining $\boldsymbol{O}$ and $\boldsymbol{A}$, the shortest ones are of length

$$
\left[\left(\theta_{1}\right)^{2}+\cdots+\left(\theta_{p}\right)^{2}+(q-p) \pi^{2}\right]^{1 / 2},
$$

where $\theta_{1}, \cdots, \theta_{p}$ are the nonzero angles between $O$ and $A$. Such a geodesic segment is called a minimal geodesic segment of the $(q-p+1)$ th type. Obviously, a minimal geodesic segment of the $k$ th type is shorter than one of the $(k+1)$ th type. A minimal geodesic segment of the first type is a minimal segment in the usual sense.

We can prove

ThEOREM 3.1. In $G_{n}\left(F^{n+m}\right)$, a point $A$ is a conjugate point to the point $O$ iff there exists a continuous family of (distinct) minimal geodesic segments of the first or the second type joining $O$ and $\boldsymbol{A}$.

Concerning first conjugate points, we can prove

TheOREM 3.2. (a) In a $G_{n}\left(R^{n+m}\right)$, any conjugate point $\boldsymbol{A}$ to the point $O$ is the first conjugate point to $O$ along some minimal geodesic segment of the first or the second type joining $O$ and $A$.

(b) In $a G_{n}\left(C^{n+m}\right)$ or $G_{n}\left(H^{n+m}\right)$, a conjugate point $\boldsymbol{A}$ to the point $\boldsymbol{O}$ either is the first conjugate point to $O$ along some minimal segment joining $O$ and $A$, or is such that the mid-point of some minimal geodesic segment $\Gamma$ of the second type joining $O$ and $A$ is the first conjugate point to $O$ along $\Gamma$.

Given two points $O$ and $A$ in $G_{n}\left(F^{n+m}\right)$, the existence or nonexistence of a continuous family of minimal geodesic segments of the first or the second type joining them and the nature of such a family if it exists depend entirely on the field $F$ and the dimensions of $A \cap O^{\perp}$ and $A \cap O$. A study of the various possibilities leads to our next theorem. We first give a definition. 
A conjugate point $A$ to the point $O$ in $G_{n}\left(F^{n+m}\right)$ is said to be of type-order $[k, h]$ if there exists a maximal continuous (then $C^{\omega}$ ) family of $\infty^{h}(h \geqq 1)$ minimal geodesic segments of the $k$ th type joining $O$ and $\boldsymbol{A}$, and $k$ is the smallest in teger possible.

It follows from Theorem 3.1 that $k=1$ or 2 .

4. Conjugate locus in $G_{n}\left(F^{n+m}\right)$. Let $O$ be any $n$-plane in $F^{n+m}$; $V_{l}, \tilde{V}_{l}$ as defined in the last paragraph of $\S 2$; and $W_{l}=V_{l} \backslash V_{l+1}$, $\tilde{W}_{l}=\tilde{V}_{l} \backslash \tilde{V}_{l+1}$. Then $O$ is a point of $G_{n}\left(F^{n+m}\right)$, and we have

THEOREM 4.1. (a) In $a G_{n}\left(R^{n+m}\right)$, the conjugate locus at the point $O$ is $V_{2} \cup \tilde{V}_{1}=\left(W_{2} \cup W_{3} \cup \cdots \cup W_{n}\right) \cup\left(\tilde{W}_{1} \cup \tilde{W}_{2} \cup \cdots \cup \tilde{W}_{n}\right)$ if $n<m$; $V_{2} \cup \tilde{V}_{2}=\left(W_{2} \cup W_{3} \cup \cdots \cup W_{n}\right) \cup\left(\tilde{W}_{2} \cup \tilde{W}_{3} \cup \cdots \cup \tilde{W}_{n}\right)$ if $n=m$; $V_{2} \cup \tilde{V}_{n-m+1}=\left(W_{2} \cup W_{3} \cup \cdots \cup W_{m}\right) \cup\left(\tilde{W}_{n-m+1} \cup \tilde{W}_{n-m+2} \cup \cdots \cup \tilde{W}_{n}\right)$

if $n>m$.

Points of $W_{l}$ and $\tilde{W}_{l} \backslash V_{2}$ are conjugate points to $O$ of type-order $\left[1, \frac{1}{2} l(l-1)\right]$ and $[2, m-n+2(l-1)]$, respectively.

(b) In a $G_{n}\left(C^{n+m}\right)$, the conjugate locus at the point $O$ is $V_{1} \cup \tilde{V}_{1}=\left(W_{1} \cup W_{2} \cup \cdots \cup W_{n}\right) \cup\left(\dot{W}_{1} \cup \tilde{W}_{2} \cup \cdots \cup \tilde{W}_{n}\right)$ if $n \leqq m$; $V_{1} \cup \tilde{V}_{n-m+1}=\left(W_{1} \cup W_{2} \cup \ldots \cup W_{m}\right) \cup\left(\tilde{W}_{n-m+1} \cup \tilde{W}_{n-m+2} \cup \ldots \cup \tilde{W}_{n}\right)$ if $n>m$.

Points of $W_{l}$ and $\tilde{W}_{l} \backslash V_{1}$ are conjugate points to $O$ of type-order $\left[1, l^{2}\right]$ and $[2,2(m-n+2 l)-3]$, respectively.

(c) In a $G_{n}\left(H^{n+m}\right)$, the conjugate locus at the point $O$ is

$$
\begin{aligned}
& V_{1} \cup \tilde{V}_{1}=\left(W_{1} \cup W_{2} \cup \ldots \cup W_{n}\right) \cup\left(\tilde{W}_{1} \cup \tilde{W}_{2} \cup \ldots \cup \tilde{W}_{n}\right) \text { if } n \leqq m ; \\
& V_{1} \cup \tilde{V}_{n-m+1}=\left(W_{1} \cup W_{2} \cup \ldots \cup W_{m}\right) \cup\left(\tilde{W}_{n-m+1} \cup \tilde{W}_{n-m+2} \cup \cdots \cup \tilde{W}_{n}\right)
\end{aligned}
$$

if $n>m$.

Points of $W_{l}$ and $\tilde{W}_{l} \backslash V_{1}$ are conjugate points to $O$ of type-order [1, $l(2 l+1)]$ and $[2,4(m-n+2 l)-5]$, respectively.

Theorem 3.2 shows that in a $G_{n}\left(R^{n+m}\right)$ the first conjugate locus coincides with the conjugate locus. It is known [14] that the minimum (or cut) locus in any $G_{n}\left(F^{n+m}\right)$ at the point $O$ is $V_{1}$. Thus it follows from Theorems 3.2 and 4.1 that in a $G_{n}\left(C^{n+m}\right)$ or $G_{n}\left(H^{n+m}\right)$ the first conjugate locus coincides with the minimum locus. This is a special case of a known result due to Crittenden [4, Theorem 5].

We conclude with two special cases of Theorem 4.1 . 
(1) The projective spaces $F P^{m}=G_{1}\left(F^{m+1}\right), m \geqq 1$. In this case, we have the following results, already known (see, for example, [2, pp. 225-226]):

\begin{tabular}{|c|c|c|}
\hline & Conjugate locus at 0 & Type-order of conjugate point \\
\hline$R P^{1}$ & Empty & \\
\hline$R P^{m}, m>1$ & $\{0\}$ & $\{0\}:[2, m-1]$ \\
\hline$C P^{m}, m \geqq 1$ & $O \perp \cup\{0\}$ & $O^{\perp}:[1,1],\{O\}:[2,2 m-1]$ \\
\hline$H P^{m}, m \geqq 1$ & $O \perp \cup\{0\}$ & $O^{\perp}:[1,3],\{O\}:[2,4 m-1]$ \\
\hline
\end{tabular}

(2) The $G_{2}\left(F^{m+2}\right), m \geqq 2$. In this case, we have

\begin{tabular}{|c|c|c|}
\hline$G_{2}\left(R^{4}\right)$ & Conjugate locus at 0 & Type-order of conjugate point \\
\hline$G_{2}\left(R^{m+2}\right), m>2$ & $\left\{O^{\perp}\right\} \cup\{\}$ & $\left\{O^{\perp}\right\}:[1,1],\{\boldsymbol{O}\}:[2,2]$ \\
\hline$G_{2}\left(C^{m+2}\right), m \geqq 2$ & $W_{2} \cup \tilde{W}_{1} \cup\{\boldsymbol{O}\}$ & $W_{2}:[1,1], \tilde{W}_{1}:[2, m-2],\{\boldsymbol{O}\}:[2, m]$ \\
\hline$W_{1} \cup W_{2} \cup \tilde{W}_{1} \cup\{\boldsymbol{O}\}$ & $\begin{array}{r}W_{1}:[1,1], W_{2}:[1,4] \\
W_{1} \backslash W_{1}:[2,2 m-3],\{\boldsymbol{O}\}:[2,2 m+1]\end{array}$ \\
\hline$G_{2}\left(H^{m+2}\right), m \geqq 2$ & $W_{1} \cup W_{2} \cup \tilde{W}_{1} \cup\{\boldsymbol{O}\}$ & $\begin{array}{r}W_{1}:[1,3], W_{2}:[1,10] \\
W_{1}:[2,4 m-5],\{\boldsymbol{O}\}:[2,4 m+3]\end{array}$ \\
\hline
\end{tabular}

where

$$
W_{1}=\left\{Z: \operatorname{dim}\left(Z \cap O^{\perp}\right)=1\right\}, \quad \tilde{W}_{1}=\{Z: \operatorname{dim}(Z \cap O)=1\}
$$

are respectively an "( $m-1)$-plane" bundle and a "line" bundle over $W_{1} \cap \widetilde{W}_{1}=F P^{1} \times F P^{m-1}$, and

$$
\begin{aligned}
& W_{2}=\left\{Z: \operatorname{dim}\left(Z \cap O^{\perp}\right)=2\right\}=\left\{O^{\perp}\right\} \quad \text { if } m=2, \\
& =G_{2}\left(F^{m}\right) \text { if } m>2 \text {. }
\end{aligned}
$$

\section{REFERENCES}

1. A. Allamigeon, Propriêtés globales des espaces de Riemann harmoniques, Ann. Inst. Fourier, Grenoble (15) 2 (1965), 91-132.

2. R. L. Bishop and R. J. Crittenden, Geometry of manifolds, Academic Press, New York, 1964.

3. S. S. Chern, Topics in differential geometry, Institute for Advanced Study lecture notes, Princeton, N. J., 1951.

4. R. Crittenden, Minimum and conjugate points in symmetric spaces, Canad. J. Math. 14 (1962), 320-328. 
5. L. W. Green, Auf Wiedersehenflachen, Ann. of Math. 78 (1963), 289-299.

6. S. Helgason, Differential geometry and symmetric spaces, Academic Press, New York, 1962.

7. W. V. D. Hodge and D. Pedoe, Methods of algebraic geometry, Vol. II, Cambridge Univ. Press, New York, 1952.

8. W. Klingenberg, Manifolds with restricted conjugate locus, Ann. of Math. 78 (1963), 527-547.

9. S. Myers, Connections between differential geometry and topology, I. Simply connected surfaces, Duke Math. J. 1 (1935), 376-391.

10. - Connections between differential geometry and topology, II. Closed surfaces, Duke Math. J. 2 (1936), 95-102.

11. H. E. Rauch, Geodesics and Jacobi equations on homogeneous Riemannian manifolds, Proceedings of U.S.-Japan Seminar in Differential Geometry, Kyoto, Japan, 1965, Tokyo (1966), pp. 115-127.

12. F. W. Warner, The conjugate locus of a Riemannian manifold, Amer. J. Math. 87 (1965), 575-604.

13. — Conjugate loci of constant order, Ann. of Math. 86 (1967), 192-212.

14. Y. C. Wong, Differential geometry of Grassmann manifolds, Proc. Nat. Acad. Sci. U.S.A. 57 (1967), 589-594.

University OF Hong Kong, Hong Kong 